\title{
Couple and family therapies for post-traumatic stress disorder (PTSD) (Protocol)
}

\author{
Cowlishaw S, Evans L, Suomi A, Rodgers B
}

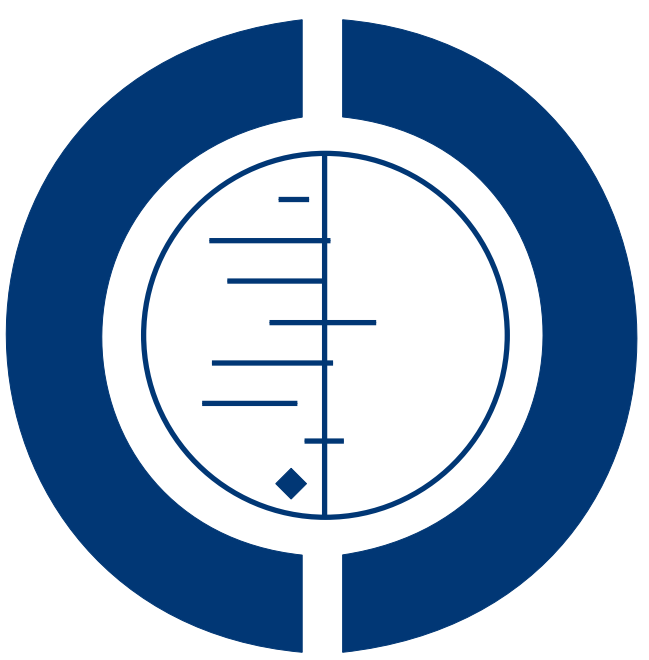

\section{THE COCHRANE COLLABORATION $^{\circledR}$}

This is a reprint of a Cochrane protocol, prepared and maintained by The Cochrane Collaboration and published in The Cochrane Library 2014, Issue 9

http://www.thecochranelibrary.com

\section{WILEY}


TABLE OF CONTENTS

HEADER . . . . . . . . . . . . . . . . . . . . . . . . . . . . . . . . . . . . 1

ABSTRACT . . . . . . . . . . . . . . . . . . . . . . . . . . . . . . . . . . . . . .

BACKGROUND . . . . . . . . . . . . . . . . . . . . . . . . . . . . . . . . . . . .

OBJECTIVES . . . . . . . . . . . . . . . . . . . . . . . . . . . . . . . . . . . . . . .

METHODS . . . . . . . . . . . . . . . . . . . . . . . . . . . . . . . . . . . . . .

ACKNOWLEDGEMENTS . . . . . . . . . . . . . . . . . . . . . . . . . . . . . . . . . . . . . . . . .

REFERENCES . . . . . . . . . . . . . . . . . . . . . . . . . . . . . . . . . . . . . . . 10

APPENDICES . . . . . . . . . . . . . . . . . . . . . . . . . . . . . . . . . . . . . 13

CONTRIBUTIONS OF AUTHORS . . . . . . . . . . . . . . . . . . . . . . . . . . . . . . . . . . . . . . . . 14

DECLARATIONS OF INTEREST . . . . . . . . . . . . . . . . . . . . . . . . . . . . . . . . 14

SOURCES OF SUPPORT . . . . . . . . . . . . . . . . . . . . . . . . . . . . . . . . . . 


\title{
[Intervention Protocol] \\ Couple and family therapies for post-traumatic stress disorder (PTSD)
}

\author{
Sean Cowlishaw ${ }^{1,2}$, Lynette Evans ${ }^{3}$, Aino Suomi ${ }^{2}$, Bryan Rodgers ${ }^{2}$ \\ ${ }^{1}$ Centre for Academic Primary Care, School of Social and Community Medicine, University of Bristol, Bristol, UK. ${ }^{2}$ School of \\ Sociology, ANU College of Arts and Social Sciences, Australian National University, Canberra, Australia. ${ }^{3}$ School of Psychological \\ Science, Faculty of Science, Technology and Engineering, La Trobe University, Melbourne, Australia \\ Contact address: Sean Cowlishaw, sean.cowlishaw@bristol.ac.uk. sean.cowlishaw@gmail.com.
}

Editorial group: Cochrane Depression, Anxiety and Neurosis Group.

Publication status and date: New, published in Issue 9, 2014.

Citation: Cowlishaw S, Evans L, Suomi A, Rodgers B. Couple and family therapies for post-traumatic stress disorder (PTSD). Cochrane Database of Systematic Reviews 2014, Issue 9. Art. No.: CD011257. DOI: 10.1002/14651858.CD011257.

Copyright (C) 2014 The Cochrane Collaboration. Published by John Wiley \& Sons, Ltd.

\begin{abstract}
A B S T R A C T
This is the protocol for a review and there is no abstract. The objectives are as follows:

The objectives of this review will be to:

1. assess the efficacy of couple and family therapies for adult PTSD, relative to 'no treatment' conditions, 'standard care', and structured or non-specific individual psychological therapies;

2. examine the clinical characteristics of studies that influence the relative efficacy of these therapies; and

3. critically evaluate methodological features of studies that bias research findings.
\end{abstract}

\section{B A C K G R O U N D}

\section{Description of the condition}

Post-traumatic stress disorder (PTSD) refers to an anxiety or trauma and stressor related disorder where symptom onset is linked to personal or vicarious exposure to traumatic events. These include events characterised by death or threatened death, sexual violence, as well as actual or threatened serious injury (American Psychiatric Association 2013). The previous fourth edition of the Diagnostic and Statistical Manual of Mental Disorders (DSM-IV TR) (American Psychiatric Association 2000), which is most commonly used in currently available research, defines three categories of psychiatric symptoms that may indicate a diagnosis of PTSD. These include:

1. intrusive re-experiencing of the event (e.g., through flashbacks and dreams);

2. avoidance of reminders and emotional numbing; and

3. persistent high levels of arousal and reactivity (e.g., hypervigilance to threat).

These symptom clusters have been re-organised in the recent fifth edition of the Diagnostic and Statistical Manual of Mental Disorders (DSM-5) (American Psychiatric Association 2013), which now identifies four categories of PTSD symptoms:

1. intrusion;

2. avoidance; 
3. negative alterations in cognitions and mood; and

4. alterations in arousal and reactivity.

The revised system thus re-positions emotional numbing in a category that also includes negative cognitions (e.g., self-blame) and emotions, while arousal symptoms are repositioned in a category including irritable and reckless or self-destructive behaviour (the latter are new symptoms). Notwithstanding these revisions, the fundamental construct built into the updated criteria is unchanged (Friedman 2011), whereby close comparability between DSM-IV and DSM-5 diagnoses is expected (Regier 2013). Current data suggest a lifetime prevalence of PTSD around $8 \%$ in the general population (Kessler 1995), and indicates a disorder that often follows a chronic course (Orcutt 2004; Solomon 2006). PTSD is also associated with a range of adverse individual outcomes (e.g., poor health, suicidality) (Sareen 2007), as well as significant interpersonal problems, including difficulties in intimate and family relationships (Taft 2011).

Most evidence linking PTSD to family problems is derived from studies of military veterans, from Europe and the United States, which document associations among post-traumatic symptoms and various adverse relationship outcomes (Galovski 2004). These include low relationship satisfaction (Goff 2007), family violence (Glenn 2002), and family members' own mental health problems (Jordan 1992). Comparative investigations of other trauma populations are relatively few, but also suggest links between PTSD and problems in intimate relationships. For example, studies following natural disasters indicate relations between post-traumatic symptoms and poor relationship adjustment (e.g., Taft 2009), while PTSD following interpersonal victimisation predicts family violence (e.g., Krause 2006). Studies of survivors of childhood sexual abuse also suggest problems with intimate relationships in adulthood (e.g., Cloitre 1997; Lamoureux 2012), including specific difficulties with intimacy and sexual dysfunction (Davis 2000). However, the unique influences of PTSD in the development of these long-term problems remain poorly understood.

The inter-relations among PTSD and family problems are likely to be complex, reflecting both the impact of post-traumatic symptoms on other family members, and effects of the family environment on PTSD. On the one hand, avoidance symptoms may reduce involvement in family activities, while emotional numbing can inhibit self-disclosure and intimacy (Erbes 2008). Hyperarousal symptoms are linked to irritability and anger and can also precipitate aggression and family conflict (Taft 2007a; Taft 2007b). On the other hand, prospective studies of veterans show that family relationships can predict change in PTSD (Evans 2009; Evans 2010), whereby an adaptive family environment can reduce the severity of symptoms, or exacerbate problems if interpersonal patterns are dysfunctional. These inter-relations are likely to be particularly complex when PTSD is linked to certain types of trauma. These may include interpersonal trauma (e.g., sexual assault), where relationships (including family relationships) are associated with the traumatic event and the onset of symptoms, as well as other events (e.g., natural disasters, motor vehicle accidents) which impact directly on multiple family members simultaneously (Riggs 2009).

\section{Description of the intervention}

Evidence of associations among post-traumatic symptoms and family difficulties has provided impetus for consideration of couple and family therapies for PTSD. General reviews of literature on couple therapies, such as Baucom 1998 and Snyder 2006, distinguish two main classes of couple-based interventions (and by extension, therapies working with broader family systems) when used for individual mental health problems. These include (1) generic therapies, developed to treat distressed relationships and address common interpersonal problems that can exacerbate individual symptoms, and (2) disorder-specific interventions, targeting interactions between interpersonal processes and specific symptoms of the disorder or its treatment.

Snyder 2006 describes several classes of generic therapies for distressed relationships that are often considered in clinical trials. First among these are behavioural therapies (e.g., traditional behavioural couple therapy) (Christensen 2004), which comprise techniques for enhancing family members' relationship skills in problem solving and communication, and increasing the frequency of positive interactions. Second are therapies based on psychodynamic and attachment theory perspectives (e.g., insight oriented marital therapy) (Snyder 1989), that are characterised by a broad focus on developing awareness and expression of unknown feelings, thoughts and needs that may underlie interpersonal patterns (Baucom 1998). Other generic therapies are also available (although considered less often in clinical trials) (Snyder 2006), and can include cognitive strategies for changing ways of thinking about behaviours and relationships, as well as techniques for enhancing emotional acceptance. Another general class of interventions may include 'systemic' therapies (Coulter 2013), potentially including structural and strategic family therapies that focus on changing patterns of family interaction and organisation (Madanes 1981; Minuchin 1974). Integrative therapies draw from multiple conceptual models (Lebow 1997).

A number of disorder-specific couple and family therapies for PTSD have also been proposed and are reviewed by Riggs 2009. They include therapies based on behavioural principles and others grounded in cognitive-behavioural models or attachment theory (Figley 1988; Johnson 1998; Monson 2004; Mueser 1995). These targeted therapies are commonly oriented towards reducing partners' distress or dysfunction in the couple relationship, as well as promoting improvements in individual PTSD. Monson 2004, for example, propose a stand-alone cognitive-behavioural treatment for post-traumatic symptoms and relationship functioning that consists of several stages of therapy. These initially deliver psycho-education about PTSD and relationship functioning, and also include behavioural interventions (e.g., communication 
skills training) to address avoidance and emotional numbing in the context of relationships. Subsequent stages comprise scheduled activities to reduce experiential avoidance and increase positive couple experiences, as well as dyadic cognitive interventions that target cognitions maintaining PTSD and relationship problems (Brown-Bowers 2012). Alternative interventions comprise adjunctive therapies that are delivered alongside other primary psychological and pharmacological treatments (Sautter 2009). Most of these interventions have been developed in the context of combatrelated PTSD (Monson 2009), with a small number (such as emotionally focused therapy) proposed originally for use with victims of sexual or physical abuse (Johnson 1998), or with traumatised populations more generally (Figley 1988).

\section{How the intervention might work}

Given the complex inter-relations among post-traumatic symptoms and family adjustment, multiple mechanisms of change may underlie the proposed effects of couple and family therapies for PTSD. For example, interventions that enhance relationship skills (e.g., problem solving, communication) can equip families to manage interpersonal difficulties (e.g., associated with avoidance of social situations) and thus minimise frustrations and family conflicts that are linked to PTSD. Therapies which promote family members' mutual understanding of post-traumatic symptoms and impacts on relationship dynamics (e.g., through increased sharing of experiences) might also assist in correcting erroneous beliefs about interpersonal behaviour (for example, a mistaken explanation for low affective involvement in terms of disengagement from the relationship, rather than emotional numbing), and further reduce family conflict. Interventions that enhance communication, or shared thoughts and feelings, may also facilitate enhanced self-disclosure and related experiences of emotional intimacy (Laurenceau 1998). These therapies will also operate through common factors shared across different interventions (e.g., positive expectancies of therapeutic change) (Sprenkle 2004), and other processes that are relatively unique to specific clinical models; for example, emotionally focused therapy, which is argued to work, in part, by accessing and reprocessing negative affect that underlies dysfunctional patterns (Johnson 1998).

Improvements in individual functioning during therapy, including reductions in post-traumatic symptoms, are also expected to involve various mechanisms. In some instances, these individual benefits may result from the reduction of significant negative exchanges in family relationships (e.g., reflecting high levels of criticism, hostility and emotional over-involvement) that can act as psychosocial stressors and exacerbate PTSD symptoms (Tarrier 1999). Conversely, couple and family therapies may also promote symptom change by enabling family members to provide both comfort and social support; the latter of which predicts positive adjustment to both physical health problems and psychological disorders like PTSD (Dirkzwager 2003; Frasure-Smith 2000; Glass
1992; Kaniasty 2008). With reference to trauma in particular, Johnson 1998 suggests that comforting and supportive relationships provide a safe and secure 'recovery environment' where individuals can reprocess and integrate traumatic memories, safely experience post-traumatic symptoms (e.g., flashbacks), and learn to regulate associated negative affective states.

\section{Why it is important to do this review}

Despite growing research on the links between PTSD and the qualities of intimate and family relationships, there remains limited understanding of the efficacy of couple and family therapies for PTSD in adults. As far as can be ascertained, there is only one existing Cochrane systematic review that has considered family-based therapies (among others) for PTSD (Gillies 2012), and this review did not consider adult samples (but rather, focused on children and adolescents). Other Cochrane reviews of interventions for PTSD in adults have considered psychological therapies (Bisson 2007), pharmacological treatments (Stein 2006), as well as combined pharmacological and psychological interventions (Hetrick 2010). None of these have considered couple or family therapies. Other relevant Cochrane reviews have focused on prevention of PTSD and treatment of distress immediately (i.e., one to three months) following trauma exposure (Roberts 2009; Rose 2002). The currently proposed review will thus provide the first focused examination of best quality clinical trials of couple and family therapies for PTSD in adults.

\section{O B J E C T I VES}

The objectives of this review will be to:

1. assess the efficacy of couple and family therapies for adult PTSD, relative to 'no treatment' conditions, 'standard care', and structured or non-specific individual psychological therapies;

2. examine the clinical characteristics of studies that influence the relative efficacy of these therapies; and

3. critically evaluate methodological features of studies that bias research findings.

\section{MET HOD S}

\section{Criteria for considering studies for this review}

\section{Types of studies}


Eligible studies will be randomised controlled trials (RCTs) of couple or family therapies for PTSD, or associated family difficulties, in adult samples. Cross-over trials are not expected in this context, but we will include them if couples or families are randomly allocated to treatment sequence. Cluster-randomised trials will also be eligible. We will not use sample size and language of the report to determine inclusion, and there will be no restrictions on the study settings that are eligible for the review. We will not consider quasi-randomised trials (using non-random forms of allocation to groups, such as sequential allocation) for inclusion.

\section{Types of participants}

Participants will be intact couples or families, comprised of family members of any ethnicity or sexual orientation, in which at least one adult family member (over the age of 18 years) meets criteria for PTSD. Consistent with Lebow 2012, we will define couples as "long-term committed unions of romantic partners whether or not these unions are recognised by the state"; thus including gay and lesbian and other long-standing relationships, irrespective of formal recognition as 'married'. We will define a family as a couple with one or more children. In all cases one adult will be identified as suffering PTSD. It will be required that participants are diagnosed with PTSD according to recognised classification systems, including the International Classification of Diseases (ICD)-10 (WHO 2010), DSM-IV and DSM-5 (American Psychiatric Association 2000; American Psychiatric Association 2013). Assessment strategies considered appropriate for ascertainment of PTSD criteria will include general clinical interviews (e.g., based on DSM criteria) and structured clinical interviews (e.g., Clinician Administered PTSD Scale) (Blake 1995). We will also consider self-report assessment tools (e.g., PTSD Checklist; Weathers 1993) with validated clinical cut-offs.

Although we will consider studies of diverse family structures, it is expected that most participants will be adult couples who are intimate partners in marital or common law relationships. Studies where intimate partners are divorced or separated will not be considered. Studies of treatments for child or adolescent PTSD, or therapies that focus mainly on family violence are also out of scope.

\section{Types of interventions}

\section{Experimental interventions}

The review will consider any type of therapy that is intended to treat intact couples or families where at least one adult family member meets criteria for PTSD. We intend to evaluate several main categories of therapies as follows.

1. Cognitive-behavioural therapies: this category of interventions will include therapies based predominantly on behavioural and cognitive-behavioural approaches to treatment
(Figley 1988; Monson 2004). Interventions based on pure cognitive approaches would also be classified under this category of therapy.

2. Psychodynamic therapies: this category of interventions will include therapies based predominantly on psychodynamic approaches to treatment. This may include emotion-focused and insight-oriented therapies (Johnson 1998; Snyder 1989).

3. Systemic therapies: this category of interventions will include therapies derived generally from general systems theory approaches to treatment (von Bertalanffy 1969). It will include structural therapies as well as strategic therapies, among others (Coulter 2013; Madanes 1981; Minuchin 1974), and interventions that draw from multiple systemic frameworks.

4. Integrative therapies: this category of interventions will include therapies that include components of treatment drawn from multiple conceptual models (Lebow 1997), including those listed above. Where potential integrative therapies are apparent, there will be initial efforts to classify the therapy as predominantly one type of treatment (where around $80 \%$ of sessions are dedicated to one component of treatment). Where it is not possible to classify one predominant type of treatment, the intervention will be classified as an integrative therapy.

We will consider additional categories of interventions as studies become available. Eligible therapies will be delivered as 'standalone' treatments, as well as 'adjunctive' therapies delivered in conjunction with other primary treatments (e.g., individual psychological therapy). We will include disorder-specific interventions developed for treatment of PTSD or associated family difficulties (Riggs 2009). We will also consider generic therapies for relationship discord that are delivered in the context of family members diagnosed with PTSD (Snyder 2006).

For the purpose of this review, it is required that interventions will be delivered by psychiatrists, psychologists, counsellors, nurses or other health professionals with specialist training in family therapy (including students under supervision). Our review scope is focused on therapies that work directly with intact couples or families and studies where patients mainly attend therapy sessions alone will not be considered.

\section{Control conditions}

The review will consider a range of control comparators including 'no treatment' controls, 'standard care', and structured or nonspecific individual psychological therapies.

For the purpose of this review, no treatment control conditions will refer mainly to wait-list and assessment only controls.

Standard care will refer to a heterogeneous category of existing treatments or clinical practices that may be non-specific and described variously as 'existing practice', 'treatment as usual' or 'usual care' (Freedland 2011). These may involve relatively rigorous conditions (e.g., standard of care). They might also comprise eclectic interventions including naturalistic prescribing of medications, or 
minor systemic components (e.g., family member psycho-education).

Structured or non-specific individual psychological therapies will include manualised individual therapies, such as those based on general approaches described in Types of interventions (e.g., cognitive-behavioural), and other therapies for PTSD (e.g., eye movement desensitisation and reprocessing) (Bisson 2007). Non-specific individual psychological therapies provide generic features of therapy, including clinical contact and human interaction (e.g., clinician warmth, empathy, social support), and a treatment rationale (Mohr 2009). As such, they may reflect practices that approximate supportive or humanistic therapy to varying degrees.

The aim of this review is not to consider the superiority of different types of couple and family therapies. As such, we will exclude comparisons among alternative couple or family therapies as well as comparisons with partial treatment controls (e.g., the same couple or family intervention, minus key components of therapy that may drive therapeutic change).

We will also exclude studies that compare a couple or family therapy with an experimental pharmacological treatment (although comparisons with individual therapies that involve naturalistic prescribing of medications will be eligible).

\section{Types of outcome measures}

The current review will consider outcomes that address multiple domains of individual, couple and family adjustment. Additional outcomes, such as marital stability and observational measures of marital interaction, as well as potential adverse events (e.g., substance abuse, self-harm) may be considered in updates as studies and data become available.

\section{Primary outcomes}

1. Severity of PTSD symptoms, as demonstrated by the primary presenting patient and ascertained using self-reports or clinician reports on measurement scales such as the PTSD Checklist (Weathers 1993), the PTSD symptom scale (Foa 1993), as well as the Clinician Administered PTSD Scale (Blake 1995); which is considered a gold standard measure in many contexts (Weathers 2001)

2. Severity of psychological symptoms of family members, ascertained using self-reports or clinician reports on measures of mental health symptom severity (e.g., PTSD Checklist; Weathers 1993) or psychological distress (e.g., the five-item Mental Health Index of the 36-item Short Form health survey (SF-36); Ware 2000). We will consider data from adult romantic partners and children in the family separately where sufficient data are available 3. Dyadic adjustment, ascertained using self-report, family member reports or clinician reports on measures of relationship satisfaction or distress, like the Dyadic Adjustment Scale or the Marital Adjustment Test (Locke 1959; Spanier 1976)

\section{Secondary outcomes}

4. Severity of co-occurring depression or anxiety, as demonstrated by the primary presenting patient and ascertained using self-reports or clinician reports on measurement scales such as the Beck Depression Inventory or the Beck Anxiety Inventory (Beck 1961; Beck 1988)

5. Overall family functioning, ascertained using self-report, family member reports or clinician reports of overall family functioning, or specific characteristics of family interaction (e.g., communication), as measured through scales like the McMaster Family Assessment Device or the Family Environment Scale (Epstein 1983; Moos 1986)

6. Treatment dropout will be used as a proxy measure of treatment acceptability, and will be defined by the proportion of participants in treatment and control conditions that provide data at the most immediate post-treatment assessment.

7. Instances of severe aggression or violence will be considered as a type of adverse event (see Christensen 2005). Other types of adverse events (e.g., substance abuse, self-harm) may be considered in updates of this review as data becomes available.

\section{Multiple informants}

When data on dyadic adjustment or family functioning are available from multiple family members (e.g., when both partners in a couple report on relationship satisfaction), we will combine data from multiple informants to make use of all available data. This will be done be calculating the simple arithmetic mean of scores (assuming that all family members provide reports on the same scale) and the pooled variance. Exceptions may be where different family members show widely divergent perspectives on relationships, as demonstrated by limited shared variance (i.e., $<50 \%$ or $r=0.70$ ). In such instances, reports from different family members may be considered in separate analyses. Assuming the most studies will not provide data on shared variance, we will examine the implications of decisions to average across multiple informants through sensitivity analyses.

\section{Timing of outcome assessment}

We will examine data from all outcomes at: (a) immediate posttreatment assessments, conducted from 0 to 3 months following the completion of therapy; and (b) follow-up assessments, conducted more than 3 months but less than 12 months following completion of therapy. We will also consider additional and longer periods of follow-up assessment if relevant data are available.

\section{Search methods for identification of studies}

We will conduct a systematic search procedure to identify all available relevant evidence. This systematic search procedure will comprise two main strategies including: (1) electronic searches of 
databases and clinical trials registries; and (2) manual searches of other resources.

\section{Electronic searches}

We will perform electronic searches of multiple databases. These databases will include the Cochrane Depression, Anxiety and Neurosis Review Group's Specialised Register (CCDANCTR), which covers relevant RCTs indexed in EMBASE (1974-), MEDLINE (1950-) and PsycINFO (1967-), as well as the Cochrane Central Register of Controlled Trials (CENTRAL, The Cochrane Library, all years). For a full description of the CCDANCTR, please see Appendix 1.

We will also conduct supplementary searches of the following additional databases:

- Literature in the Health Sciences in Latin America and the Caribbean (LILACS);

- Published International Literature on Traumatic Stress (PILOTS); and

- Web of Science.

We will search the CCDANCTR (Studies and References Registers) using the following free-text terms:

- (PTSD or post-trauma* or *trauma* or "stress disorder*" or (combat and disorder*) or (war and neuro*)) AND (couple* or partner* or marriage or marital or husband* or wife or wives* ${ }^{*}$ or spous* or family or families or multi-family or conjoint or interpersonal or relations* or (child* and parent*)) AND (*therap* ${ }^{*}$ or counsel* ${ }^{*}$ or treat* or intervention*).

We will adapt these search terms to conduct analogous searches of additional databases (e.g., PILOTS). We will apply no date or language restrictions. We will also search the World Health Organization (WHO) International Clinical Trials Registry Platform (ICTRP) search portal (http://apps.who.int/trialsearch/) and ClinicalTrials.gov (http://clinicaltrials.gov/) to identify unpublished and/or ongoing studies.

\section{Searching other resources}

\section{Handsearching}

We will manually search the early editions of key journals to identify potentially relevant studies that may not be indexed in the databases. These key journals will include:

- Journal of Traumatic Stress (1988 - 2000);

- Journal of Family Psychology (1987 - 2000); and

- Journal of Marital and Family Therapy (1980 - 2000).

\section{Reference lists}

We will also manually screen the reference lists and bibliographies of all included studies to identify other relevant references.

\section{Data collection and analysis}

\section{Selection of studies}

We will select studies in stages. First, we will screen the titles and abstracts (where available) of all records retrieved to determine potentially eligible studies. Two review authors will screen each record. We will obtain full-text articles of any studies that would appear to meet inclusion criteria, as well as those that cannot be excluded based on title and abstract, for further assessment. Two review authors will independently examine each full-text article in order to confirm eligibility, and disagreement will be resolved through discussion. We will identify any duplicate publications and list them along with the primary publication. We will record and present decisions made during the study selection process, as well as the names and numbers of studies and reasons for exclusion at each stage, in a Preferred Reporting Items for Systematic Reviews and Meta-Analyses (PRISMA) flow diagram.

\section{Data extraction and management}

Following the identification of eligible studies, we will extract data on study characteristics from reports using a piloted, structured data extraction template. Data extraction will endeavour to obtain information (where available) relating to publication details (e.g., country of origin, year of publication), sample characteristics (e.g., age and ethnicity of participants, predominant type of trauma), clinical characteristics (e.g., type of therapy, duration of treatment), methodology (e.g., inclusion/exclusion criteria, timing of follow-up assessments), statistical analyses and results (e.g., strategies for managing non-independent data from family members, group means and standard deviations for primary and secondary outcomes). Two review authors will independently extract data from each study to ensure accuracy.

\section{Main comparisons}

Multiple comparisons are planned to evaluate the efficacy of standalone couple or family therapies for PTSD compared to relevant control comparators. These include:

1. couple or family therapy versus no treatment;

2. couple or family therapy versus standard care; and

3. couple or family therapy versus structured or non-specific individual psychological therapy.

Additional comparisons are planned to evaluate the efficacy of adjunctive couple or family therapies, additional to primary treatment, relative to controls. These include:

1. couple or family therapy (adjunctive to standard care) versus standard care alone;

2. couple or family therapy (adjunctive to structured or nonspecific individual psychological therapies) versus structured or non-specific individual psychological therapies alone. 
Additional types of comparisons may be considered as studies become available.

Comparisons involving adjunctive therapies will be limited to control conditions that involve substantively similar primary treatments. As such, we will not consider comparisons between couple or family therapies adjunctive to primary treatment and (a) 'no treatment' controls, and (b) substantively different primary treatments (e.g., cognitive-behavioural therapy versus psychodynamic individual therapy). Where multiple couple or family therapy conditions are compared with control conditions, it is envisaged that the couple or family therapy conditions will be combined (Unit of analysis issues).

\section{Assessment of risk of bias in included studies}

Two review authors will independently assess risk of bias associated with each study. Both authors will allocate a judgement of 'High', 'Low' or 'Unclear' risk of bias with regard to several design characteristics that are among the main sources of bias in clinical trials (Higgins 2011b). Disagreements between review authors with regard to classification of studies will be resolved through discussion. In line with available recommendations (Juni 1999), we will assess each source of bias independently.

\section{Random allocation to groups (sequence generation)}

It is an eligibility requirement that studies use random allocation to groups. Notwithstanding this, it is envisaged that the level of detail published about randomisation procedures may vary. We will classify studies which provide limited or no detail about randomisation as having unclear risk of bias.

\section{Allocation concealment \\ Adequate concealment of allocation requires that participants and researchers are kept unaware, and are unable to foresee, the groups to which participants are allocated (Schulz 2002). We will classify studies that lack allocation concealment as having high risk of bias.}

\section{Blinding}

Blinding can refer to hiding the nature of the intervention delivered from multiple potential groups (e.g., participants, treatment providers, outcome assessors) (Montori 2002), and we will consider the following blinding aspects.

1. Participants and treatment providers: blinding of participants and treatment providers is usually easy to accomplish in studies of pharmacological treatments, but it is rarely feasible for psychological therapies. Accordingly, it is expected that most studies will be classified as having a high risk of bias.

2. Outcome assessors: blinding of outcome assessment will refer to masking of group allocation from outcome assessors (e.g., researchers administering symptom scales). Studies that fail to blind outcome assessors (including studies relying on self-report measures completed by participants) will be classified as having a high risk of bias. Given that blinding of outcomes assessors may vary within studies and across outcomes (e.g., some may be selfreported with other outcomes evaluated using blinded outcome assessors), this characteristic will be assessed separately for each outcome considered in Types of outcome measures.

\section{Incomplete outcome data}

According to Higgins 2011b, missing data can be caused by both study exclusions and attrition. Justifiable reasons for exclusions may include identifying (after randomisation) that participants were ineligible for the study. In contrast, participants may be excluded because they did not receive the intended intervention in accordance with the protocol (or for other reasons), which may lead to bias (Higgins 2011b). In case of missing data from attrition, primary studies may report analyses conducted using data from participants providing complete information (i.e., 'completers only'), or by including data from all participants through use of various missing data strategies. These include recommended strategies based on principles of maximum-likelihood or multiple imputation, as well as older (and potentially biased) forms of imputation including mean imputation and last observation carried forward (LOCF) (Graham 2009).

For the purpose of this review, we will classify studies as having a high risk of bias if they violate any of three principles of intentionto-treat (ITT) analyses described by Higgins 2011b. These are:

1. "keep participants in the intervention groups to which they were randomised, regardless of the intervention they received";

2. "measure outcome data on all participants"; and

3. "include all randomised participants in the analyses".

Given that approaches to managing incomplete outcome data (from attrition in particular) may vary within studies and across outcomes, we will assess these approaches separately for each outcome considered in Types of outcome measures.

\section{Selective outcome reporting}

Selective outcome reporting refers to the presentation of a limited subset of data or analyses based on the nature (e.g., statistical significance) of results (Hutton 2000). Although there are various issues suggestive of selective outcome reporting (Higgins 2011b), we will classify studies in this review as having high risk of bias if they have protocols or entries in trial registries that list primary or secondary outcomes that differ from those reported in the published results (lacking credible explanation). We will classify studies that are not associated with published protocols or adequately detailed entries in trial registries as having an unclear risk of bias.

\section{Measures of treatment effect}




\section{Dichotomous data}

For evaluation of treatment effects based on dichotomous outcomes (e.g., scores in the clinically significant range on relationship adjustment), we will use the risk ratios (RRs) and associated $95 \%$ confidence intervals (CIs).

\section{Continuous data}

For evaluation of treatment effects based on continuous outcomes we will use the mean differences (MDs), where outcomes are reported on the same scale, or the standardised mean difference (SMDs) where outcomes are reported on different scales. We will obtain SMDs by calculating the difference between raw means and dividing by the pooled variance of treatment and control conditions. We will present 95\% CIs around the MDs or SMDs.

\section{Unit of analysis issues}

\section{Cluster-randomised trials}

Where a cluster-randomised trial is identified, we will extract the methods used to analyse data, while the inflated standard error approach will be used to adjust standard errors for non-independence of observations (Higgins 2011c). To facilitate this, we will extract the degree of non-independence, as reflected in the intraclass correlation (ICC). Where the ICC is not reported, a value of 0.05 will be assumed.

\section{Cross-over trials}

Where a cross-over trial is identified, we will consider data from the between-group comparison from the first treatment stage only.

\section{Studies with multiple treatment groups}

Where multiple couple or family therapy conditions are compared with a 'no treatment' or individual intervention control, we will combine the couple or family therapy conditions using the formulae reported by Higgins 2011a. Exceptions may be where a standalone couple or family therapy and an adjunctive therapy (alongside another primary treatment) are both compared with an individual therapy condition, and where the adjunctive condition provides a significant additional dosage of therapy (in terms of number of sessions). Rather, we will evaluate stand-alone and adjunctive therapy conditions in separate comparisons (Data extraction and management). Where different groups are involved in the same treatment, but have results reported separately, we will also combine these data.

\section{Dealing with missing data}

\section{Missing information about study design and results/statistics}

Information about research design that is not reported in a primary publication will be initially ascertained through examination of duplicate publications. Where informative duplicate publications are unavailable, and where missing data relate to the inclusion criteria or risk of bias (as defined in this review), we will contact the study authors for additional information. We will also seek clarification from the study authors where statistics necessary for the estimation of treatment effects (e.g., standard deviations) are missing.

\section{Missing observations from primary studies due to attrition}

Our decision to consider 'completers only' data or data from all participants will be initially determined by the type of information reported; for example, if the study only reports analyses of the 'completers only' sample. However, preference will be given to data from all randomised participants (where available). Given certain 'old' missing data strategies (such as mean or single imputation or LOCF) that may still introduce bias into the study (Graham 2009), we will examine these through sensitivity analyses.

\section{Assessment of heterogeneity}

\section{Clinical heterogeneity}

For studies that are clinically heterogeneous or present insufficient information to facilitate quantitative synthesis, we will present a narrative summary of results.

\section{Statistical heterogeneity}

We will assess statistical heterogeneity across studies using the $\mathrm{I}^{2}$ statistic, which indicates the percentage of total variability across studies that is due to between-study differences (Huedo-Medina 2006). We will also examine the $\mathrm{Chi}^{2}$ statistic and associated significance test (P value). However, this statistic lacks power to detect true differences (Deeks 2011), and greater emphasis will thus be placed on $\mathrm{I}^{2}$.

Although thresholds for $\mathrm{I}^{2}$ are arbitrary, there are overlapping bands that may suggest minor $(0 \%$ to $40 \%)$, moderate $(30 \%$ to $60 \%$ ), substantial ( $50 \%$ to $90 \%$ ), and considerable (75\% to $100 \%)$ levels of heterogeneity (Deeks 2011). Interpretation of the $\mathrm{I}^{2}$ statistic will be qualified through evaluation of the pattern of variability, and whether all studies indicate beneficial effects of treatment. Where strong evidence of true heterogeneity is present, the pooled effect will be considered as a limited, though 'best available' estimate of the expected magnitude of the treatment effect. 


\section{Assessment of reporting biases}

We will examine multiple databases to identify published research, while trial registers will be searched to identify unpublished studies. We will use funnel plots and the linear regression test to evaluate publication bias where there are more than $k=10$ studies available (Egger 1997: Sterne 2011). We will also screen relevant databases and trial registers to identify reports published in a nonEnglish language.

\section{Data synthesis}

Two authors will enter data into the Cochrane Collaboration statistical software, Review Manager 2014, and we will employ the random-effects model to provide a weighted estimate of the efficacy of each intervention relative to control. This random-effects model assumes true variability in effect sizes across studies, and estimates both the average effect and degree of variability across studies (Normand 1999). Where there is evidence of true heterogeneity, it may be inappropriate to place inordinate emphasis on a weighted mean effect size (especially if some studies indicate harmful effects) and we will instead interpret the pooled estimates through discussion of statistical diversity of studies.

\section{Subgroup analysis and investigation of heterogeneity}

In the case of observed statistical heterogeneity, and where sufficient studies are available, we will pursue subgroup analyses to examine factors explaining between-study variability. We will evaluate potential differences in treatment effects according to the following study characteristics.

1. Disorder-specific versus generic couple or family therapies: Disorder-specific and generic therapies share a focus on improved relationship outcomes. However, disorder-specific therapies may include additional components of treatment targeting individual psychopathology, and may thus have greater impacts on individual post-traumatic symptoms. The more singular focus of generic therapies on relationship problems may lead to larger improvements in couple and family adjustment.

2. Nature of trauma linked to disorder onset: Patients exposed to interpersonal trauma (e.g., sexual assault) may demonstrate greater severity of problems in couple and family functioning, relative to traumas that do not have equivalent interpersonal components (e.g., combat exposure, natural disasters).

Accordingly, disorders associated with interpersonal trauma may benefit more from couple and family therapies.

3. Recent onset versus chronic PTSD: Disorders with recent onset (e.g., within one year of trauma exposure) may be more amenable to change following couple and family therapies for PTSD, relative to longer-standing conditions where symptoms and interpersonal patterns have become established over time.
We will conduct the above subgroup analyses using the approach described by Deeks 2011, applying the test for subgroup differences available in Review Manager 2014. We may consider other potential clinical characteristics (e.g., couple versus family-based therapies for PTSD) in updates as studies and literature becomes available.

\section{Sensitivity analysis}

We will conduct sensitivity analyses to examine whether findings are robust to approaches adopted in this review (Deeks 2011). We will consider the following characteristics of assumptions sequentially for the purposes of these analyses.

- Where outcome data from multiple informants are available, we will exclude data from family members.

- We will exclude cluster randomised trials.

- We will vary the ICC used during analyses of cluster randomised trials.

- We will exclude cross-over trials.

- Results based on 'completers only' will be excluded.

- Results based on imputed values for missing data will be imputed.

\section{Summary of findings table}

Summary of findings tables will be developed to summarise the key findings of the review, for all relevant populations, in line with Schünemann 2011. The tables will present findings relating to each type of intervention in terms of primary and secondary outcomes (Types of outcome measures), standardised effect size estimates (and 95\% CIs) to illustrate comparative risk, the number of studies and participants, and the quality of evidence based on standards of the Grading of Recommendations Assessment, Development and Evaluation (GRADE) working group (Balshem 2011).

\section{ACKNOWLEDGEMENTS}

\section{Funding}

The National Institute for Health Research (NIHR) is the largest single funder of the Cochrane Depression, Anxiety and Neurosis Group.

\section{Disclaimer}

The views and opinions expressed therein are those of the authors and do not necessarily reflect those of the NIHR, NHS or the Department of Health. 


\section{R E F E R E N C E S}

\section{Additional references}

American Psychiatric Association 2000

American Psychiatric Association. Diagnostic and Statistical Manual of Mental Disorders (DSM-IV). Text

Revision. Fourth. Washington, DC: American Psychiatric Association, 2000

American Psychiatric Association 2013

American Psychiatric Association. Diagnostic and Statistical Manual of Mental Disorders, Fifth Edition, DSM-5. 2013. Available from http://dsm.psychiatryonline.org/book.aspx? bookid $=556$. Arlington, VA.

Balshem 2011

Balshem H, Helfand M, Schünemann HJ, Oxman AD, Kunz R, Brozek J, et al.GRADE guidelines: 3. Rating the quality of evidence. Journal of Clinical Epidemiology 2011; 64(4):401-6.

\section{Baucom 1998}

Baucom DH, Shoham V, Mueser KT, Daiuto AD, Stickle TR. Empirically supported couple and family interventions for marital distress and adult mental health problems. Journal of Consulting and Clinical Psychology 1998;66(1): 53-88.

\section{Beck 1961}

Beck AT, Ward CH, Mendelson M, Mock J, Erbaugh J. An inventory for measuring depression. Archives of General Psychiatry 1961;4(6):561-71.

\section{Beck 1988}

Beck AT, Epstein N, Brown G, Steer RA. An inventory for measuring clinical anxiety: Psychometric properties. Journal of Consulting and Clinical Psychology 1988;56(6):893-7.

Bisson 2007

Bisson J, Andrew M. Psychological treatment of posttraumatic stress disorder (PTSD). Cochrane Database of Systematic Reviews 2007, Issue 3. [DOI: 10.1002/ 14651858.CD003388.pub3]

Blake 1995

Blake DD, Weathers FW, Nagy LM, Kaloupek DG, Gusman FD, Charney DS, et al.The development of a clinician-administered PTSD scale. Journal of Traumatic Stress 1995;8:79-90.

Brown-Bowers 2012

Brown-Bowers A, Fredman SJ, Wanklyn SG, Monson CM. Cognitive-behavioral conjoint therapy for posttraumatic stress disorder: Applications to a couple's shared traumatic experience. Journal of Clinical Psychology 2012;68(5): 536-47.

\section{Christensen 2004}

Christensen A, Atkins DC, Berns S, Wheeler J, Baucom DH, Simpson LE. Traditional versus integrative behavioral couple therapy for significantly and chronically distressed married couples. Journal of Consulting and Clinical Psychology 2004;72(2):176-91.

\section{Christensen 2005}

Christensen A, Baucom DH, Vu CT, Stanton S. Methodologically sound, cost-effective research on the outcome of couple therapy. Journal of Family Psychology 2005;19:6-17.

\section{Cloitre 1997}

Cloitre M, Scarvalone P, Difede J. Posttraumatic stress disorder, self- and interpersonal dysfunction among sexually retraumatized women. Journal of Traumatic Stress 1997;10: 437-52.

\section{Coulter 2013}

Coulter S. Systemic psychotherapy as an intervention for post-traumatic stress responses: An introduction, theoretical rationale and overview of developments in an emerging field of interest. Journal of Family Therapy 2013;35:381-406.

\section{Davis 2000}

Davis JL, Petretic-Jackson PA. The impact of child sexual abuse on adult interpersonal functioning: A review and synthesis of the empirical literature. Aggression and Violent Behavior 2000;5:291-328.

\section{Deeks 2011}

Deeks JJ, Higgins JPT, Altman DG (editors). Chapter 9: Analysing data and undertaking meta-analyses. In: Higgins JPT, Green S (editors). Cochrane Handbook for Systematic Reviews of Interventions Version 5.1.0 (updated March 2011). The Cochrane Collaboration, 2011. Available from www.cochrane-handbook.org.

\section{Dirkzwager 2003}

Dirkzwager AJE, Bramsen I, van der Ploeg HM. Social support, coping, life events, and posttraumatic stress symptoms among former peacekeepers: A prospective study. Personality and Individual Differences 2003;34:1545-59.

Egger 1997

Egger M, Smith GD, Minder C. Bias in meta-analysis detected by a simple, graphical test. BMJ 1997;315:629.

Epstein 1983 Epstein NB, Baldwin LM, Bishop DS. The McMaster Family Assessment Device. Journal of Marital and Family Therapy 1983;9:171-80.

Erbes 2008

Erbes CR, Polusny MA, MacDermin S, Compton JS. Couple therapy with combat veterans and their partners. Journal of Clinical Psychology 2008;64:972-83.

Evans 2009

Evans L, Cowlishaw S, Hopwood M. Family functioning predicts outcomes for veterans in treatment for chronic posttraumatic stress disorder. Journal of Family Psychology 2009;23:531-9.

Evans 2010

Evans L, Cowlishaw S, Forbes D, Parslow R, Lewis V. Longitudinal analyses of family functioning in veterans and their partners across treatment. Journal of Consulting and Clinical Psychology 2010;78:611-22. 
Figley 1988

Figley CR. A five-phase treatment of post-traumatic stress disorder in families. Journal of Traumatic Stress 1988;1: $127-41$.

Foa 1993

Foa EB, Riggs DS, Dancu CV, Rothbaum BO. Reliability and validity of a brief instrument for assessing posttraumatic stress disorder. Journal of Traumatic Stress 1993;6: 459-73.

\section{Frasure-Smith 2000}

Frasure-Smith N, Lesperance F, Gravel G, Masson A, Juneau $\mathrm{M}$, Talajic M, et al.Social support, depression, and mortality during the first year after myocardial infarction. Circulation 2000;101:1919-24.

\section{Freedland 2011}

Freedland KE, Mohr DC, Davidson KW, Schwartz JE. Usual and unusual care: Existing practice control groups in randomized controlled trials of behavioral interventions. Psychosomatic Medicine 2011;73(4):323-35.

\section{Friedman 2011}

Friedman MJ, Resick PA, Bryant RA, Brewin CR. Considering PTSD for DSM-5. Depression and Anxiety 2011;28:750-69.

\section{Galovski 2004}

Galovski T, Lyons JA. Psychological sequelae of combat violence: A review of the impact of PTSD on the veteran's family and possible interventions. Aggression and Violent Behavior 2004;9:477-501.

Gillies 2012

Gillies D, Taylor F, Gray C, O’Brien L, D'Abrew N. Psychological therapies for the treatment of post-traumatic stress disorder in children and adolescents. Cochrane Database of Systematic Reviews 2012, Issue 12. [DOI: 10.1002/14651858.CD006726.pub2]

Glass 1992

Glass TA, Maddox GL. The quality and quantity of social support: Stroke recovery as psycho-social transition. Social Science \& Medicine 1992;34:1249-61.

\section{Glenn 2002}

Glenn DM, Beckham JC, Feldman ME, Kirby AC, Hertzberg MA, Moore SD. Violence and hostility among families of Vietnam veterans with combat-related posttraumatic stress disorder. Violence and Victims 2002;17: 473-89.

Goff 2007

Goff BSN, Crow JR, Reisbig AMJ, Hamilton S. The impact of individual trauma symptoms of deployed soldiers on relationship satisfaction. Journal of Family Psychology 2007; 21:344-53.

Graham 2009

Graham JW. Missing data analysis: Making it work in the real world. Annual Review of Psychology 2009;60:549-79.

\section{Hetrick 2010}

Hetrick SE, Purcell R, Garner B, Parslow R. Combined pharmacotherapy and psychological therapies for post traumatic stress disorder (PTSD). Cochrane Database of Systematic Reviews 2010, Issue 7. [DOI: 10.1002/ 14651858.CD007316.pub2]

\section{Higgins 2011a}

Higgins JPT, Deeks JJ (editors). Chapter 7: Selecting studies and collecting data. In: Higgins JPT, Green

S (editors), Cochrane Handbook for Systematic Reviews of Interventions Version 5.1.0 (updated March 2011). The Cochrane Collaboration, 2011. Available from www.cochrane-handbook.org.

\section{Higgins 2011b}

Higgins JPT, Altman DG, Sterne JAC (editors). Chapter 8: Assessing risk of bias in included studies. In: Higgins JPT, Green S (editors). Cochrane Handbook for Systematic Reviews of Interventions Version 5.1.0 (updated March 2011). The Cochrane Collaboration, 2011. Available from www.cochrane-handbook.org.

\section{Higgins 2011c}

Higgins JPT, Deeks JJ, Altman DG (editors). Chapter 16: Special topics in statistics. In: Higgins JPT, Green S (editors), Cochrane Handbook for Systematic Reviews of Interventions Version 5.1.0 (updated March 2011). The Cochrane Collaboration, 2011. Available from www.cochrane-handbook.org.

\section{Huedo-Medina 2006}

Huedo-Medina TB, Sánchez-Meca J, Marín-Martínez F, Botella J. Assessing heterogeneity in meta-analysis: Q statistic or I2 index?. Psychological Methods 2006;11: 193-206.

\section{Hutton 2000}

Hutton JL, Williamson PR. Bias in meta-analysis due to outcome variable selection within studies. Journal of the Royal Statistical Society Series C 2000;49:359-70.

\section{Johnson 1998}

Johnson SM, Williams-Keeler L. Creating healing relationships for couples dealing with trauma: The use of emotionally focused marital therapy. Journal of Marital and Family Therapy 1998;24:25-40.

\section{Jordan 1992}

Jordan KB, Marmar CR, Fairbank JA, Schlenger WE, Kulka RA, Hough RL, et al.Problems in families of male Vietnam veterans with posttraumatic stress disorder. Journal of Consulting and Clinical Psychology 1992;60:916-26.

\section{Juni 1999}

Juni P, Witschi A, Bloch R, Egger M. The hazards of scoring the quality of clinical trials for meta-analysis. Journal of the American Medical Association 1999;282:1054-60.

\section{Kaniasty 2008}

Kaniasty K, Norris FH. Longitudinal linkages between perceived social support and posttraumatic stress symptoms: Sequential roles of social causation and social selection. Journal of Traumatic Stress 2008;21:274-81. 


\section{Kessler 1995}

Kessler RC, Sonnega A, Bromet E, Hughes M, Nelson CB. Posttraumatic stress disorders in the National Comorbidity Survey. Archives of General Psychiatry 1995;52:1048-60.

Krause 2006

Krause ED, Kaltman S, Goodman L, Dutton MA. Role of distinct PTSD symptoms in intimate partner reabuse: A prospective study. Journal of Traumatic Stress 2006;19: 507-16.

Lamoureux 2012

Lamoureux BE, Palmieri PA, Jackson AP, Hobfoll SE. Child sexual abuse and adulthood-interpersonal outcomes: Examining pathways for intervention. Psychological Trauma: Theory, Research, Practice, and Policy 2012;4:605-13.

\section{Laurenceau 1998}

Laurenceau J, Barrett LF, Pietromonaco PR. Intimacy as an interpersonal process: The importance of self-disclosure, partner disclosure, and perceived partner responsiveness in interpersonal exchanges. Journal of Personality and Social Psychology 1998;74:1238-51.

\section{Lebow 1997}

Lebow J. The integrative revolution in couple and family therapy. Family Process 1997;36(1):1-17.

\section{Lebow 2012}

Lebow JL, Chambers AL, Christensen A, Johnson SM. Research on the treatment of couple distress. Journal of Marital and Family Therapy 2012;38(1):145-68.

\section{Locke 1959}

Locke HJ, Wallace KM. Short marital adjustment and prediction tests: Their reliability and validity. Marriage and Family Living 1959;21:251-5.

\section{Madanes 1981}

Madanes C. Strategic Family Therapy. Sanfrancisco, CA: Jossey-Bass Inc, 1981.

\section{Minuchin 1974}

Minuchin S. Families and Family Therapy. Cambridge, MA: Harvard University Press, 1974.

\section{Mohr 2009}

Mohr DC, Spring B, Freedland KE, Beckner V, Arean P, Hollon SD, et al.The selection and design of control conditions for randomized controlled trials of psychological interventions. Psychotherapy and Psychosomatics 2009;78: $275-84$.

Monson 2004

Monson CM, Schnurr PP, Stevens SP, Guthrie KA. Cognitive-behavioral couple's treatment for posttraumatic stress disorder: Initial findings. Journal of Traumatic Stress 2004;17:341-4.

Monson 2009

Monson CM, Taft CT, Fredman SJ. Military-related PTSD and intimate relationships: From description to theorydriven research and intervention development. Clinical Psychology Review 2009;29:707-14.

\section{Montori 2002}

Montori VM, Bhandari M, Devereaux PJ, Manns BJ, Ghali WA, Guyatt GH. In the dark: The reporting of blinding status in randomized controlled trials. Journal of Clinical Epidemiology 2002;55:787-90.

Moos 1986

Moos R, Moos B. The Family Environment Scale manual. 2nd Edition. Palo Alto: Consulting Psychologists Press, 1986.

\section{Mueser 1995}

Mueser KT, Glynn SM. Behavioral family therapy for psychiatric disorders. Boston: Allyn \& Bacon, 1995.

\section{Normand 1999}

Normand ST. Meta-analysis: Formulating, evaluating, combining, and reporting. Statistics in Medicine 1999;18: 321-59.

\section{Orcutt 2004}

Orcutt HK, Erickson DJ, Wolfe J. The course of PTSD symptoms among Gulf War veterans: A growth mixture modeling approach. Journal of Traumatic Stress 2004;17: 195-202.

\section{Regier 2013}

Regier DA, Narrow WE, Clarke DE, Kraemer HC, Kuramoto SJ, Kuhl EA, et al.DSM-5 field trials in the United States and Canada, part II: Test-retest reliability of selected categorical diagnoses. American Journal of Psychiatry 2013;170:59-70.

\section{Review Manager 2014}

The Nordic Cochrane Centre, The Cochrane Collaboration. Review Manager (RevMan). 5.3. Copenhagen: The Nordic Cochrane Centre, The Cochrane Collaboration, 2014.

\section{Riggs 2009}

Riggs DS, Monson CM, Glynn S, Canterino J. Couples and family therapy. In: Foa EB, Keane TM, Friedman MJ editor(s). Effective treatments for PTSD: Practice guidelines from the International Society for Traumatic Stress Studies. 2nd Edition. New York: Guilford, 2009:458-478.

\section{Roberts 2009}

Roberts NP, Kitchiner NJ, Kenardy J, Bisson JI. Multiple session early psychological interventions for the prevention of post-traumatic stress disorder. Cochrane Database of Systematic Reviews 2009, Issue 3. [DOI: 10.1002/ 14651858.CD006869.pub2]

\section{Rose 2002}

Rose SC, Bisson J, Churchill R, Wessely S. Psychological debriefing for preventing post traumatic stress disorder (PTSD). Cochrane Database of Systematic Reviews 2002, Issue 2. [DOI: 10.1002/14651858.CD000560]

\section{Sareen 2007}

Sareen J, Cox BJ, Stein MB, Afifi TO, Fleet C, Asmundson GJG. Physicla and mental comorbidity, disability, and suicidal behavior associated with Posttraumatic Stress Disorder in a large community sample. Psychosomatic Medicine 2007;69:242-8. 


\section{Sautter 2009}

Sautter FJ, Glynn SM, Thompson KE, Franklin L, Han $\mathrm{X}$. A couple-based approach to the reduction of PTSD avoidance symptoms: Preliminary findings. Journal of Marital and Family Therapy 2009;35:343-9.

\section{Schulz 2002}

Schulz KF, Grimes DA. Allocation concealment in randomised trials: Defending against deciphering. Lancet 2002;359(9306):614-8.

\section{Schünemann 2011}

Schünemann HJ, Oxman AD, Higgins JPT, Vist GE, Glasziou P, Guyatt GH. Chapter 11: Presenting results and 'Summary of findings' tables. In: Higgins JPT, Green S (editors), Cochrane Handbook for Systematic Reviews of Interventions Version 5.1.0 (updated March 2011). The Cochrane Collaboration, 2011. Available from www.cochrane-handbook.org.

\section{Snyder 1989}

Snyder DK, Wills RM. Behavioral versus insight-oriented marital therapy: Effects on individual and interspousal functioning. Journal of Consulting and Clinical Psychology 1989;57:39-46.

\section{Snyder 2006}

Snyder DK, Castellani AM, Whisman MA. Current status and future directions in couple therapy. Annual Review of Psychology 2006;57:317-44.

\section{Solomon 2006}

Solomon Z, Mikulincer M. Trajectories of PTSD: A 20-year longitudinal study. American Journal of Psychiatry 2006; 163:659-66.

\section{Spanier 1976}

Spanier GB. Measuring dyadic adjustment: New scale for assessing the quality of marriage and similar dyads. Journal of Marriage and the Family 1976;38:15-28.

\section{Sprenkle 2004}

Sprenkle DH, Blow AJ. Common factors and our sacred models. Journal of Marital and Family Therapy 2004;30: 113-29.

Stein 2006

Stein DJ, Ipser JC, Seedat S. Pharmacotherapy for post traumatic stress disorder (PTSD). Cochrane Database of Systematic Reviews 2006, Issue 1. [DOI: 10.1002/ 14651858.CD002795.pub2]

Sterne 2011

Sterne JAC, Egger M, Moher D (editors). Chapter 10: Addressing reporting biases. In: Higgins JPT, Green $S$ (editors). Cochrane Handbook for Systematic Reviews of Intervention. Version 5.1.0 (updated March 2011). The Cochrane Collaboration, 2011. Available from www.cochrane-handbook.org.

\section{Taft 2007a}

Taft CT, Kaloupek DG, Schumm JA, Marshall AD, Panuzio J, King DW, et al.Posttraumatic stress disorder symptoms, physiological reactivity, alcohol problems, and aggression among military veterans. Journal of Abnormal Psychology 2007;116:498-507.

\section{Taft 2007b}

Taft CT, Street AE, Marshall AD, Dowdall DJ, Riggs DS. Posttraumatic stress disorder, anger, and partner abuse among Vietnam combat veterans. Journal of Family Psychology 2007;2:270-7.

\section{Taft 2009}

Taft CT, Monson CM, Schumm JA, Watkins LE, Panuzio J, Resick PA. Posttraumatic Stress Disorder symptoms, relationship adjustment, and relationship aggression in a sample of female flood victims. Journal of Family Violence 2009;24:389-96.

Taft 2011

Taft CT, Watkins LE, Stafford J, Street AE, Monson CM. Posttraumatic stress disorder and intimate relationship problems: A meta-analysis. Journal of Consulting and Clinical Psychology 2011;79:22-33.

\section{Tarrier 1999}

Tarrier N, Sommerfield C, Pilgrim H. Relatives' expressed emotion (EE) and PTSD treatment outcome. Psychological Medicine 1999;29:801-11.

von Bertalanffy 1969

von Bertalanffy L. General System Theory: Foundations, Development, Applications. New York: George Brazillier, Inc, 1969.

\section{Ware 2000}

Ware J, Kosinski M, Gandek B. Overview of the SF-36. SF36 health survey: Manual and interpretation guide. Lincoln: Quality Metric Incorporated, 2000.

\section{Weathers 1993}

Weathers FW, Litz BT, Herman DS, Huska JA, Keane TM. The PTSD Checklist (PCL): Reliability, Validity, and Diagnostic Utility. Paper presented at the 9th Annual Conference of the International Society for Traumatic Stress Studies (ISTSS), San Antonio, TX, October, 1993. Available from http://www.pdhealth.mil/library/downloads/ pcl_sychometrics.doc.

\section{Weathers 2001}

Weathers FW, Keane TM, Davidson JRT. Clinicianadministered PTSD scale: A review of the first ten years of research. Depression and Anxiety 2001;13(3):132-56.

\section{WHO 2010}

World Health Organization. International Statistical Classification of Diseases and Related Health Problems 10th Revision. 2010. Available from http://apps.who.int/ classifications/icd10/browse/2010/en.

* Indicates the major publication for the study 


\section{A P P E N D I C E S}

\section{Appendix I. Further information on CCDANCTR}

The Cochrane Depression, Anxiety and Neurosis Group (CCDAN) maintain two clinical trials registers at their editorial base in Bristol, UK, a references register and a studies based register. The CCDANCTR-References Register contains over 33,000 reports of randomised controlled trials in depression, anxiety and neurosis. Approximately $60 \%$ of these references have been tagged to individual, coded trials. The coded trials are held in the CCDANCTR-Studies Register and records are linked between the two registers through the use of unique Study ID tags. Coding of trials is based on the EU-Psi coding manual. Please contact the CCDAN Trials Search Coordinator for further details. Reports of trials for inclusion in the Group's registers are collated from routine (weekly), generic searches of MEDLINE (1950-), EMBASE (1974-) and PsycINFO (1967-); quarterly searches of the Cochrane Central Register of Controlled Trials (CENTRAL) and review specific searches of additional databases. Reports of trials are also retrieved from WHO ICTRP search portal, ClinicalTrials.gov, drug companies, the hand-searching of key journals, conference proceedings and other (nonCochrane) systematic reviews and meta-analyses. Details of CCDAN's generic search strategies can be found on the Group's website. The CCDANCTR is hosted and maintained on the new Cochrane Register of Studies (CRS) meta-register, which allows for left- and right-hand truncation of search terms.

\section{CONTRIBUTIONS OFAUTHORS}

Sean Cowlishaw conceptualised the review. Sean Cowlishaw, Lynette Evans, Aino Suomi and Bryan Rodgers all contributed to the development of the protocol. All authors will contribute to the conduct and writing of the review.

\section{DECLARATIONSOF INTEREST}

The authors have no conflicts of interest to declare.

\section{SOURCESOFS UPPORT}

\section{Internal sources}

- University of Bristol, UK.

Salary support (SC)

- La Trobe University, Australia.

Salary support (LE)

- Australian National University, Australia.

Salary support (AS, BR)

\section{External sources}

- National Health \& Medical Research Council, Australia.

BR receives salary support from National Health \& Medical Research Council (Fellowship No. 471429). 